\title{
Reduction of Aerobic Acetate Production by Escherichia coli
}

\author{
WILLIAM R. FARMER AND JAMES C. LIAO* \\ Department of Chemical Engineering, Texas A\&M University, College Station, Texas 77843-3122
}

Received 22 November 1996/Accepted 13 May 1997

\begin{abstract}
Acetate excretion by Escherichia coli during aerobic growth on glucose is a major obstacle to enhanced recombinant protein production. We report here that the fraction of carbon flux through the anaplerotic pathways is one of the factors influencing acetate excretion. Flux analysis of $E$. coli central metabolic pathways predicts that increasing the fraction of carbon flux through the phosphoenolpyruvate carboxylase (PPC) pathway and the glyoxylate bypass reduces acetate production. We tested this prediction by overexpressing PPC and deregulating the glyoxylate bypass by using a $f a d R$ strain. Results show that the acetate yield by the fadR strain with PPC overexpression is decreased more than fourfold compared to the control, while the biomass yield is relatively unaffected. Apparently, the fraction of carbon flux through the anaplerotic pathways is one of the factors that influence acetate excretion. These results confirm the prediction of our flux analysis and further suggest that $\boldsymbol{E}$. coli is not fully optimized for efficient utilization of glucose.
\end{abstract}

Escherichia coli, like other facultative anaerobes, can derive ATP from either aerobic respiration or substrate level phosphorylation such as acetate production. Obviously, the former is much more efficient than the latter. However, it is commonly observed that $E$. coli excretes 10 to $30 \%$ of carbon flux from glucose to acetate in glucose-containing media even when the culture is fully aerated. Acetate excretion is a major limitation in batch fermentations and leads to decreased recombinant protein yields $(21,28,32)$. Different strategies have been applied to prevent the accumulation of acetate in the medium. At the process level, algorithms have been developed to control glucose concentration in bioreactors (18) and to add glucose according to complex feeding schedules in fed-batch fermentations $(17,30,33)$. The purpose of these techniques is to balance glucose feeding with consumption and to prevent oxygen limitations at higher cell densities. Since acetate is produced mainly through the actions of phosphotransacetylase (encoded by pta) and acetate kinase (encoded by $a c k$ ), genetic techniques have been applied to obtain pta mutants, which showed decreased acetate production (2). However, in some cases, the pta knockout mutation leads to high pyruvate production, which is also undesirable (9). Construction of mutants with modified glucose uptake rates (6) and channeling of carbon flux away from acetate production towards other end products $(1,2,7,9)$ have been reported in the literature.

Aerobic acetate production is a manifestation of the imbalance between glucose uptake and the demands for both biosynthesis and energy production (11). However, the cause of such an imbalance is largely unknown. The most common arguments are that the glucose uptake rate is improperly controlled and that the activity of the tricarboxylic acid (TCA) cycle is limiting. Substrates or mutations that reduce glycolytic flux apparently also reduce acetate production, although at the expense of the specific growth rate (14). In addition, Holms (14) reported that the flux through citrate synthase, the first enzyme in the TCA cycle, is lower in E. coli grown on glucose than in the same strain grown on fructose, a substrate that does not lead to acetate excretion.

To investigate this problem, we have performed a mathe-

\footnotetext{
* Corresponding author. Present address: Department of Chemical Engineering, University of California-Los Angeles, Los Angeles, CA 90024. Phone: (310) 825-2046. Fax: (310) 206-4107.
}

matical analysis of the distribution of metabolic fluxes in E. coli (8) based on the stoichiometry of central metabolism. This analysis predicts that increasing the relative flux (normalized by glucose uptake) through the anaplerotic reactions would reduce acetate production by $E$. coli in minimal media containing glucose.

Two possible anaplerotic sequences that can be used when E. coli is grown on glucose are the phosphoenolpyruvate (PEP) carboxylase (PPC) reaction and the glyoxylate bypass (Fig. 1a). However, the former is highly regulated (29) while the latter cannot be effectively induced in the presence of glucose (20). $\mathrm{PPC}$ is a tetrameric enzyme which is activated by fructose-1,6diphosphate (FDP), acetyl coenzyme A (acetyl-CoA), GTP, and guanosine tetraphosphate (ppGpp) and is inhibited by aspartate and malate (29). The glyoxylate bypass contains two enzymes, isocitrate lyase (ICL) (ace $A$ product) and malate synthase ( $a c e B$ product), which are located on the aceBAK operon, with $a c e K$ coding for isocitrate dehydrogenase kinase/ phosphatase. The transcriptional regulation of this operon involves many factors, including IclR $(3,24,35)$, FadR $(23-25)$, FruR (5), and ArcAB $(15,16)$. The expression of aceBAK is induced during growth on acetate or fatty acids, but induction is prevented in the presence of glucose, glycerol, or pyruvate (20) or under anaerobic conditions. In addition to transcriptional control, the flux through the glyoxylate bypass is also regulated at the branch point of isocitrate, where isocitrate dehydrogenase and ICL compete for the same substrate (22).

Because of the complex regulation acting on these pathways, changes in genomic structure have to be implemented to elevate the fluxes through these anaplerotic sequences. In this study, we overexpressed PPC from a multicopy plasmid and derepressed the glyoxylate pathway by introducing a fadR::Tn10 insertional mutation. Our results show that a significant reduction in acetate production is achieved in both exponential and stationary phases. This metabolic engineering approach improves growth characteristics without having to use complex control algorithms and feeding schedules to reduce acetate production.

\section{MATERIALS AND METHODS}

Materials. D-Glucose, isopropyl- $\beta$-D-thiogalactopyranoside (IPTG), and all of the other chemicals used were purchased from Sigma Chemical Co. (St. Louis, Mo.). Total protein assay reagent was purchased from Bio-Rad (Hercules, Calif.). 

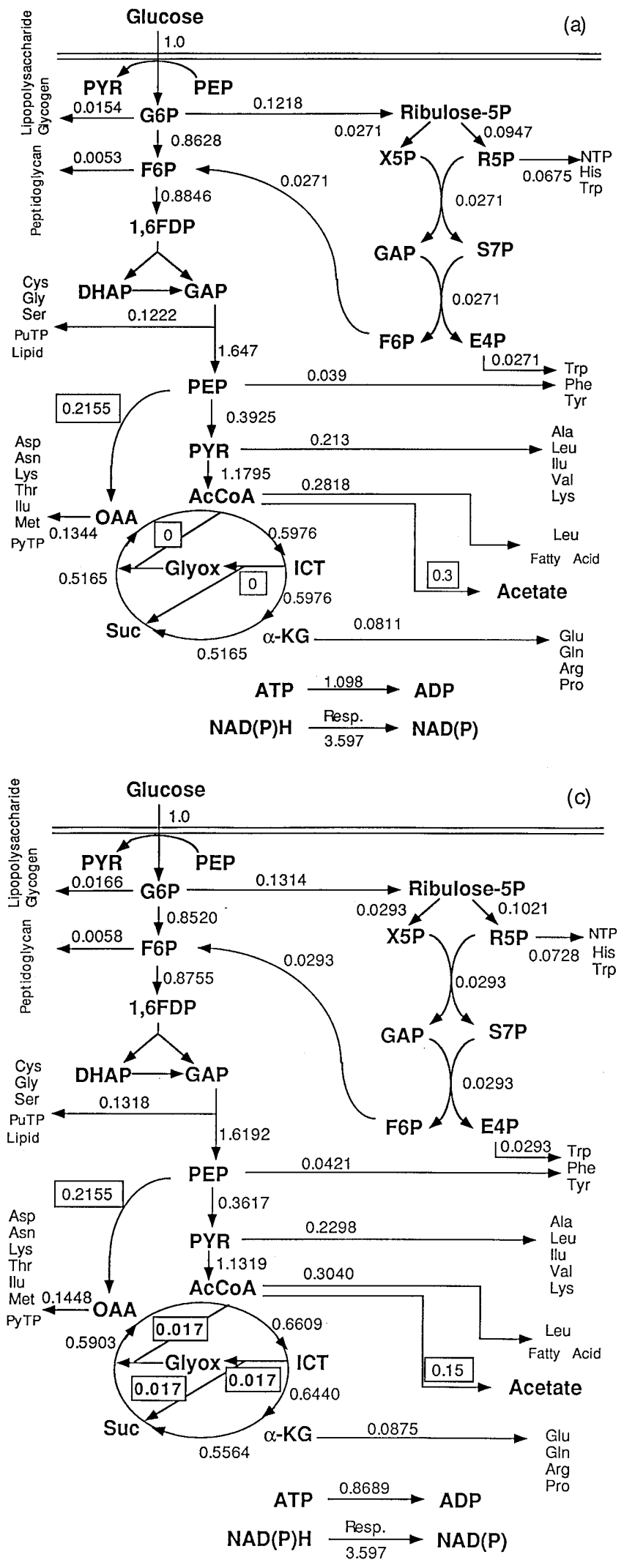

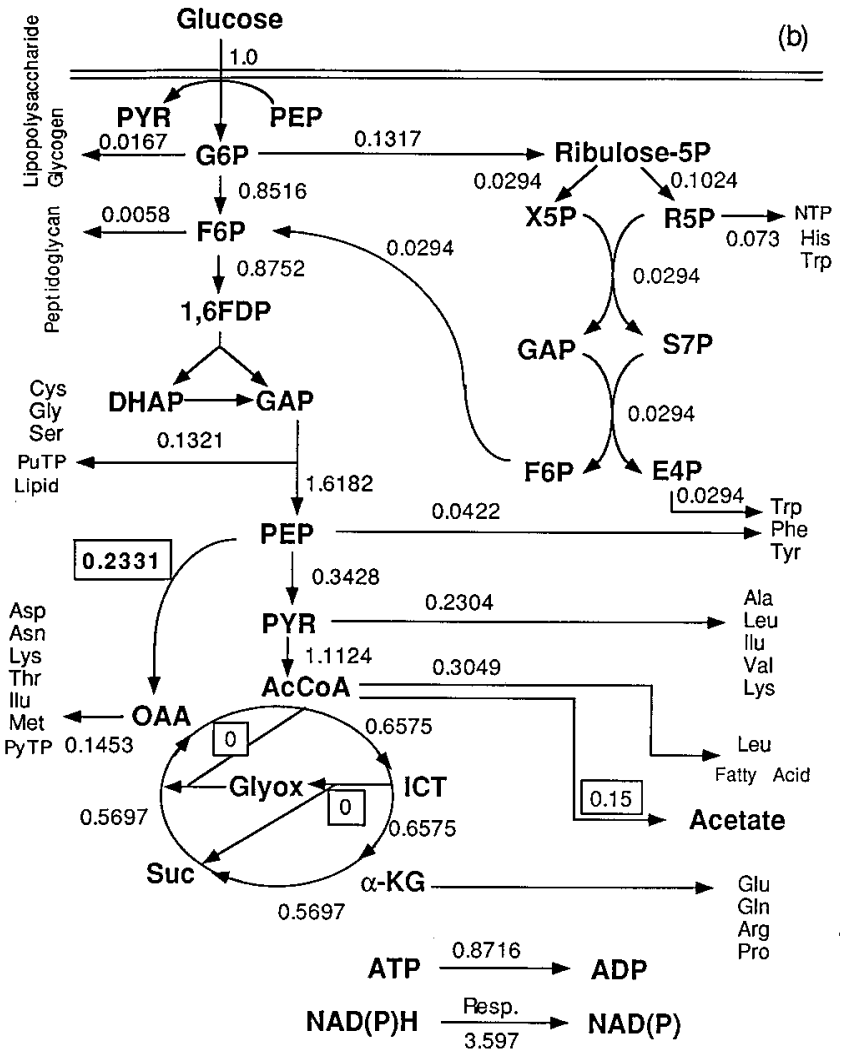

FIG. 1. Fluxes that are boxed are of direct relevance to this study. (a) Relative flux distribution in a wild-type E. coli strain, in which the fluxes were calculated based on typical experimental yields of biomass, acetate, and $\mathrm{CO}_{2}$ and normalized by the glucose consumption. The flux through the glyoxylate bypass was assumed to be zero. (b) Relative flux distribution of an E. coli strain that overexpresses PPC. The glucose consumption and respiration fluxes were held at the same level as in part a, while the PPC flux (in boldface) was specified at a value $8 \%$ higher than that calculated in part a. The rest of the fluxes were calculated based on these three specified fluxes. The flux through the glyoxylate bypass was again assumed to be zero. (c) Relative flux distribution in an E. coli strain with deregulated glyoxylate bypass. The glucose consumption, respiration, and PPC fluxes were held at the same level as in part a, while the glyoxylate bypass flux (in boldface) was specified at a value (0.017) such that the acetate flux was $50 \%$ of the control flux shown in part a. The calculation method was described in detail by Delgado and Liao (8). PYR, pyruvate; G6P, glucose 6-phosphate; Ribulose-5P, ribulose 5-phosphate; R5P, ribose 5-phosphate; X5P, xylulose 5-phosphate; NTP, nucleoside triphosphates; GAP, glyceraldehyde 3phosphate; S7P, sedoheptulose 7-phosphate; F6P, fructose 6-phosphate; E4P, erythrose 4-phosphate; 1,6FDP, fructose 1,6-diphosphate; DHAP, dihydroxyacetone phosphate; OAA, oxaloacetate; ICT, isocitrate; Suc, succinate; $\alpha-\mathrm{KG}$, $\alpha$-ketoglutarate.

Bacterial strains and plasmids. The E. coli strains used are listed in Table 1. Wild-type $\mathrm{F}^{-} \lambda^{-}$E. coli K-12 strain VJS632 was a generous gift from Valley Stewart, Cornell University. fadR::Tn10 E. coli K-12 strain CEM62 was kindly provided by Stan Maloy, University of Illinois, Urbana-Champaign (25). Strain JCL1225, a derivative of VJS632, was made $f a d R:: T n 10$ by P1 transduction with CEM62 as the donor with $\mathrm{Tet}^{\mathrm{r}}$ selection. The resulting strain was designated JCL1535.

Plasmid pJF118EH (12) was obtained from Michael Bagdasarian of Michigan State University. This vector is derived from pBR322 and contains $\mathrm{P}_{t a c}$ and the lacI gene. Plasmid pPC201 (4) is derived from pJF118EH with the $p p c$ gene inserted into the EcoRI site. The $p p c$ gene was cloned by PCR and contains its natural ribosome binding site but not the native promoter. Thus, the expression of $p p c$ is controlled by $\mathrm{P}_{t a c}$ on the vector.

Media and growth conditions. For strain construction, cells were grown in Luria-Bertani medium (27). For physiological characterizations, all strains were cultured in M9 minimal medium (27) with $0.5 \%$ glucose in shake flasks with a gyration rate of $250 \mathrm{rpm}$. IPTG was added $1 \mathrm{~h}$ after inoculation to $50 \mu \mathrm{M}$. Measurements began after two to three generations, when the cell densities were ca. $2 \times 10^{7} \mathrm{cells} / \mathrm{ml}$ (optical density at $550 \mathrm{~nm}\left[\mathrm{OD}_{550}\right], 0.1$ ). All cultures were 
grown at $37^{\circ} \mathrm{C}$, and ampicillin $(100 \mu \mathrm{g} / \mathrm{ml})$ was added as a selection pressure for plasmid-bearing strains. Cell growth was monitored by taking samples every 30 min and measuring the turbidity at $550 \mathrm{~nm}$ in a spectrophotometer (DU-640; Beckman). To achieve balanced growth, the shake flask cultures were monitored to mid-exponential phase (about $0.5 \mathrm{U}$ of $\mathrm{OD}_{550}$ ) and diluted to about $0.1 \mathrm{U}$ of $\mathrm{OD}_{550}$ in fresh medium containing IPTG. The diluted cultures achieved at least $3 \mathrm{~h}$ of exponential growth before transition into stationary phase. They were sampled periodically for analysis. Three independent colonies for each strain were grown and characterized, and the results were averaged.

Measurement of fermentation products and glucose. Samples for metabolite measurements were taken every hour and spun down in a centrifuge (5415C; Eppendorf). The supernatant was retained and stored at $-80^{\circ} \mathrm{C}$ until assayed. The glucose concentration in the medium was measured by use of the 3,5dinitrosalicylic acid (DNS) assay for total reducing sugars as described previously $(26,31)$. Essentially, $0.25 \mathrm{ml}$ of the sample and $0.75 \mathrm{ml}$ of the DNS reagent mixture were mixed together in a test tube. The mixtures were placed in a boiling water bath for $15 \mathrm{~min}$ and then diluted with $4 \mathrm{ml}$ of distilled water. The $A_{550}$ of the samples was measured spectrophotometrically, and the glucose concentration was calculated from a standard curve. The DNS reagent mixture was prepared by dissolving $10.6 \mathrm{~g}$ of DNS and $19.8 \mathrm{~g}$ of $\mathrm{NaOH}$ in 1.4 liters of distilled water. We subsequently added $306 \mathrm{~g}$ of sodium potassium tartrate, $7.6 \mathrm{ml}$ of phenol, and $8.3 \mathrm{~g}$ of sodium metabisulfite to the mixture. The $\mathrm{pH}$ was adjusted to 12.6 , and distilled water was added to give a final volume of 1.5 liters.

Fermentation product concentrations were measured by high-pressure liquid chromatography over an organic acid column (Aminex HPX-87H; Bio-Rad) with $0.01 \mathrm{~N}$ sulfuric acid as the eluent and a flow rate of $0.6 \mathrm{ml} / \mathrm{min}$. The column temperature was maintained at $60^{\circ} \mathrm{C}$, and the peaks were detected by determining UV $A_{210}$.

Enzyme assays. Cell extracts were prepared by disrupting cells at $1,000 \mathrm{lb} / \mathrm{in}^{2}$ in a French cell press (SLM Instruments, Inc.) (13). PPC activity was measured as described by Morikawa et al. (29). The PPC assay mixture consisted of the following reagents in a final volume of $1.5 \mathrm{ml}$ : HEPES-KOH buffer ( $\mathrm{pH} 7.3$ ), 150 mmol; $\mathrm{MgSO}_{4}, 75 \mathrm{mmol} ; \mathrm{KHCO}_{3}, 75 \mathrm{mmol}$; PEP, $0.45 \mathrm{mmol}$; acetyl-CoA, 0.525 mmol; FDP, $2.85 \mathrm{mmol}$; L-aspartate, $2.1 \mathrm{mmol}$; L-malate, $1.35 \mathrm{mmol}$; NADH, $0.45 \mathrm{mmol}$; malate dehydrogenase, $5 \mathrm{U}$. The reaction was started with addition of the cell extract. PPC activity was monitored by measuring the rate of NADH oxidation at $340 \mathrm{~nm}$. An extinction coefficient of $6,260 \mathrm{M}^{-1} \mathrm{~cm}^{-1}$ was used to convert absorption to concentration.

ICL activity was modified from Dixon and Kornberg $(10,19)$. The ICL assay mixture consisted of the following reagents in a final volume of $1.5 \mathrm{ml}$; phosphate buffer ( $\mathrm{pH} 7.4$ ), $96.8 \mathrm{mmol}$; isocitrate, $15 \mathrm{mmol} ; \mathrm{MgCl}_{2}, 15 \mathrm{mmol}$; cysteine- $\mathrm{HCl}$, $7.5 \mathrm{mmol}$; phenylhydrazine- $\mathrm{HCl}, 15 \mathrm{mmol}$. A molar extinction coefficient of $1.7 \times 10^{4} \mathrm{M}^{-1} \mathrm{~cm}^{-1}$ was used.

Total protein content was determined with the Bio-Rad dye reagent (Bradford assay) with bovine serum albumin as the protein standard.

\section{RESULTS}

Overexpression of PPC reduces acetate excretion. The strains used in this study are listed in Table 1 . To test the effect of PPC overexpression, we transformed plasmid pPC201 and the vector, pJF118EH, into JCL1225. The plasmid-containing strains were cultured in M9-glucose (5 g/liter) medium (27) with IPTG $(50 \mu \mathrm{M})$ in shake flasks. Figure $2 \mathrm{a}$ shows that JCL1225/pPC201 and the vector control (JCL1225/pJF118EH) grew at about the same rate. Overexpression of PPC did not affect cell growth significantly. However, overexpression of PPC in JCL1225/pPC201 resulted in a $60 \%$ decrease in the final acetate concentration compared to the vector control (Fig. 2b). The acetate yield on glucose $\left(Y_{A / G}\right.$, grams of acetate produced/grams of glucose consumed) was reduced twofold compared to that of JCL1225/pJF118EH, as shown in Table 2. These results are consistent with the theoretical prediction based on stoichiometry and flux balance (8). Apparently, overexpressed PPC diverts carbon flux away from acetate production towards biomass production, as observed by the simultaneous increase and decrease of the biomass yield $\left(Y_{X / G}\right.$, grams of biomass produced/grams of glucose consumed) and the acetate yield, respectively.

Deregulation of glyoxylate bypass reduces acetate production. In addition to PPC, another anaplerotic sequence that can be used in the presence of glucose is the glyoxylate bypass. To derepress the $a c e B A K$ operon, we introduced a $f a d R:: \operatorname{Tn} 10$ allele into the wild-type strain and repeated the above experiment. Indeed, acetate production in the fadR strain (JCL1535/ (a)
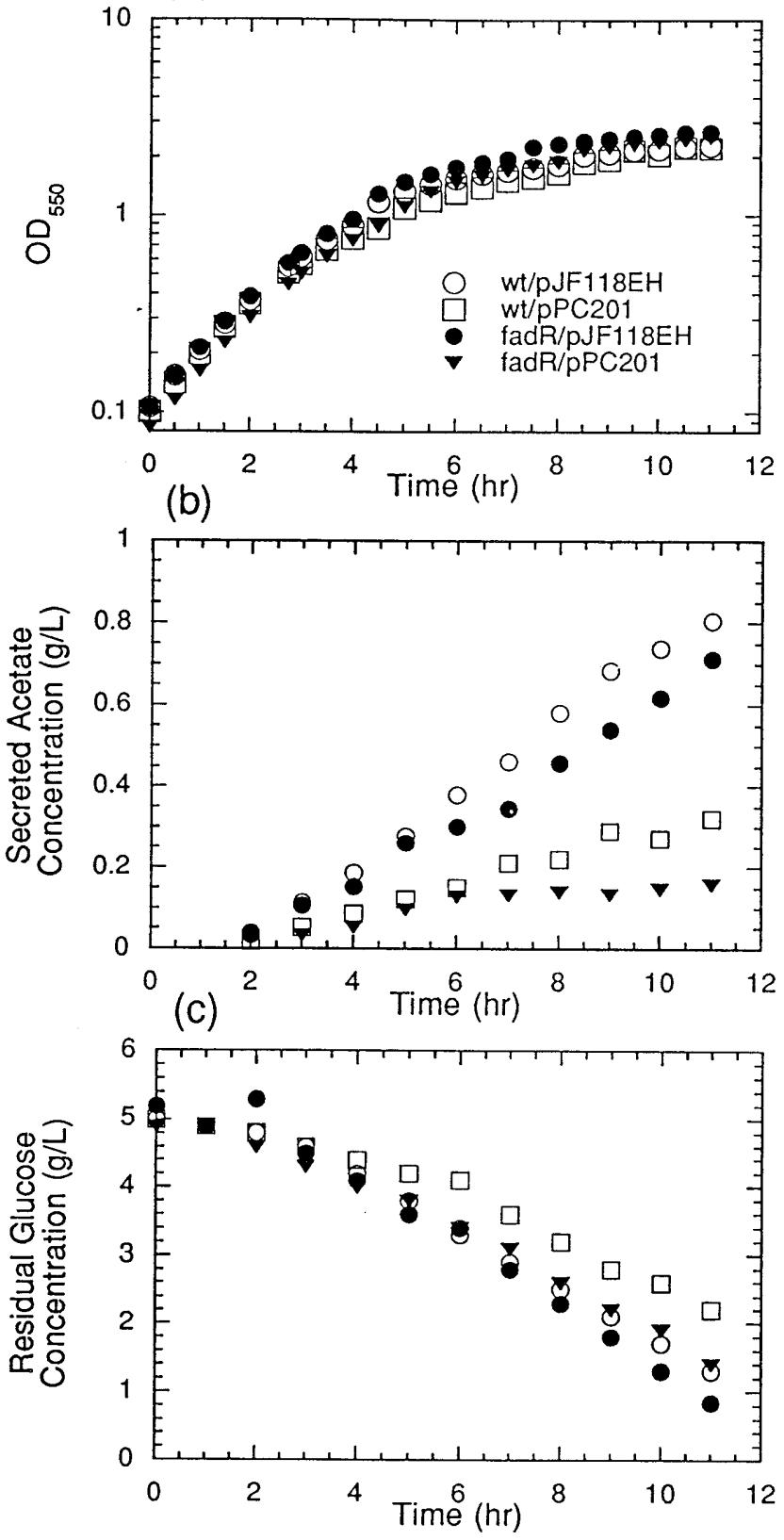

FIG. 2. Characterization of the effect of PPC overexpression and the fadR::Tn10 insertional mutation on E. coli in shake flasks with glucose at $5 \mathrm{~g} /$ liter. Cells were grown to mid-log phase and then diluted into fresh medium of the same composition. The wild-type (wt) and fadR designations represent JCL1225 and JCL1535, respectively. (a) Growth characterization of the PPC-overexpressing and fadR::Tn10 strains. (b) Time profiles of acetate production. (c) Time profiles of residual glucose concentration in the medium.

pJF118EH) is reduced, as seen by the $13 \%$ decrease in final acetate concentration compared to the $\mathrm{fadR}^{+}$control (JCL1225/ pJF118EH) in Fig. 2b. $Y_{A / G}$ for JCL1535/pJF118EH decreased about $32 \%$ without affecting $Y_{X / G}$. Specific glucose consumption by JCL1535/pJF118EH was slightly increased during balanced growth compared to the $\mathrm{fadR}^{+}$control, JCL1225/ pJF118EH. Additionally, as seen in Fig. 2a, the final cell concentration of JCL1535/pJF118EH increased by about $15 \%$. 
TABLE 1. E. coli strains and plasmids used in this study

\begin{tabular}{|c|c|c|}
\hline $\begin{array}{l}\text { Strain or } \\
\text { plasmid }\end{array}$ & Relevant characteristics & Source (reference \\
\hline \multicolumn{3}{|l|}{ Strains } \\
\hline VJS632 & $\begin{array}{l}\text { Wild-type E. coli K-12 but } \\
\mathrm{F}^{-} \lambda^{-}\end{array}$ & V. J. Stewart \\
\hline CEM62 & $\begin{array}{l}\text { Wild-type } E \text {. coli } \mathrm{K}-12 \text { but } \\
\mathrm{F}^{-} \lambda^{-} \text {fadR::Tn } 10\end{array}$ & S. Maloy (25) \\
\hline JCL1225 & $\begin{array}{l}\text { Same as VJS632 but } l a c I^{\mathrm{q}} \\
\quad \text { lacZ::Tn5 }\end{array}$ & Laboratory collection \\
\hline JCL1535 & $\begin{array}{l}\text { Same as JCL1225 but } \\
\quad \text { fadR::Tn10 }\end{array}$ & This study \\
\hline \multicolumn{3}{|l|}{ Plasmids } \\
\hline pJF118EH & $b l a^{+} l a c I^{\mathrm{q}} \mathrm{P}_{t a c}$ & J. P. Furste et al. (12) \\
\hline pPC201 & Same as pJF118EH but $p p c^{+}$ & Y. P. Chao and J. C. Liao (4) \\
\hline
\end{tabular}

These trends may reflect increased acetyl-CoA utilization by this strain during the later stages of growth. Concomitant with the increased specific glucose uptake during exponential phase, the fadR strain (JCL1535/pJF118EH) grew slightly faster than the isogenic wild-type control (JCL1225/pJF118EH).

Simultaneous overexpression of PPC and deregulation of glyoxylate bypass lead to an increased effect. As demonstrated above, overexpressing PPC or derepressing the glyoxylate bypass led to decreased acetate production. Thus, we predicted that simultaneously introducing the two genetic alterations would further reduce acetate production. Indeed, overexpressing PPC in the fadR strain (JCL1535/pPC201) led to a fivefold decrease in the final acetate concentration compared to the wild-type control (JCL1225/pJF118EH), as shown in Fig. 2b. $Y_{A / G}$ for JCL1535/pPC201 decreased by approximately $70 \%$, while $Y_{X / G}$ was only slightly decreased, as seen in Table 2.

Elevated PPC and ICL activities are concomitant with decreased acetate production. PPC activity in the crude extract of JCL1225/pPC201 was measured to correlate the PPC activity with the physiological effect. The PPC activity was about 210 $\mathrm{nmol} / \mathrm{min} / \mathrm{mg}$ of protein after induction with $50 \mu \mathrm{M}$ IPTG. The corresponding activity in the control strain, JCL1225/ pJF118EH, was approximately $33 \mathrm{nmol} / \mathrm{min} / \mathrm{mg}$ of protein, as shown in Table 3. All PPC activity assays were performed in triplicate. PPC activity in the pPC201-containing strains increased sevenfold after induction.

The activity of ICL was measured to confirm the derepression of glyoxylate bypass enzymes by the $f a d R:: T n 10$ allele. ICL activities from JCL1225 and JCL1535 with and without PPC overexpression were measured at 3 and $11 \mathrm{~h}$ after inoculation. As shown in Table 3, ICL activity was approximately fourfold higher in the $f a d R$ strain, JCL1535, than in the $f a d R^{+}$control, JCL1225, even at $3 \mathrm{~h}$, when the cultures were in exponential phase. At $11 \mathrm{~h}, \mathrm{ICL}$ activity remained about four- to fivefold higher in the $\operatorname{fadR}$ strains, which corresponds well to the decrease in acetate production observed at stationary phase.
Therefore, the reduction of acetate production can be attributed to the increased anaplerotic activity caused by overexpressed PPC and/or a derepressed glyoxylate bypass.

Production of pyruvate and succinate is negligible. Genetic techniques have been applied to obtain pta mutants which are deficient in phosphotransacetylase activity, one of the enzymes of the acetate pathway. It has been reported that the pta mutation reduces acetate excretion, although the production of pyruvate is increased dramatically (9). Therefore, we measured the concentrations of various organic acids in the medium in order to determine if the production of other fermentation byproducts increased due to the alteration of carbon flux distribution. Pyruvate and succinate concentrations are reported in Table 4. Lactate was undetectable in the medium. No substantial increase in pyruvate was observed, which implies that the pyruvate balance is unaffected. The succinate concentration was increased twofold in the strains containing the fadR locus, presumably due to the increased activity of the glyoxylate bypass.

\section{DISCUSSION}

Our group has performed a mathematical analysis of the distribution of metabolic fluxes in $E$. coli (8) based on the stoichiometry of central metabolism. The flux through the reaction is defined as the amount of product formed per unit of time per unit of cell mass (moles per gram of cell mass per hour), which is equivalent to the specific reaction rate. However, flux analysis based on stoichiometry is often discussed in terms of relative fluxes $(34,36)$, which are fluxes normalized to the glucose uptake flux. Therefore, the relative flux is dimensionless and represents the fraction of carbon intake through the particular reaction specified. Our analysis takes into account the demands for precursor metabolites for growth and the balance of fluxes at each junction of the metabolic pathways. For the purpose of conceptual illustration, we consider only the synthesis of new cell mass, excretion of acetate, and evolution of $\mathrm{CO}_{2}$ as the outlets of carbon. The analysis shows that increasing the anaplerotic flux relative to the glucose uptake would increase the relative flux to (or the yield of) biomass formation, which, in turn, increases the demand for glucose 6-phosphate, fructose 6-phosphate, ribose 5-phosphate, $\mathrm{PEP}$, and pyruvate for biosynthesis. Because of the diversion of all these metabolites to biosynthesis, the acetate excretion is greatly decreased.

This prediction is illustrated in Fig. 1a to c. Figure 1a shows the relative flux distribution of an $E$. coli strain that gives typical yields (grams per gram of glucose consumed) for acetate, $\mathrm{CO}_{2}$, and cell mass. The internal fluxes were calculated by use of experimental data for the fluxes of glucose consumption, $\mathrm{CO}_{2}$ production, acetate excretion, and cell mass formation. The calculated internal fluxes were then normalized by the glucose consumption flux to give the relative fluxes, which are shown in Fig. 1a. It is important to recognize that the stoichi-

TABLE 2. Balanced growth characteristics of strains in glucose minimal medium ${ }^{a}$

\begin{tabular}{llcccc}
\hline Strain & Plasmid & $\begin{array}{c}\text { Specific growth } \\
\text { rate }\left(\mathrm{h}^{-1}\right)\end{array}$ & $\begin{array}{c}Y_{X / G}(\mathrm{~g} \text { of dry cell wt/g } \\
\text { of glucose })\end{array}$ & $\begin{array}{c}Y_{A / G}(\mathrm{~g} \text { of acetate/g } \\
\text { of glucose })\end{array}$ & $\begin{array}{c}\text { Specific glucose consumption rate } \\
(\mathrm{g} \text { of glucose/h/g of dry cell wt) }\end{array}$ \\
\hline JCL1225 & pJF118 & 0.52 & 0.30 & 0.25 & 1.7 \\
JCL1225 & pPC201 & 0.57 & 0.35 & 0.14 & 1.6 \\
JCL1535 & pJF118 & 0.52 & 0.27 & 0.17 & 1.9 \\
JCL1535 & pPC201 & 0.51 & 0.25 & 0.076 & 2.0 \\
\hline
\end{tabular}

${ }^{a}$ Samples $(1 \mathrm{ml})$ were taken every hour and analyzed. All measurements are in triplicate and independent. Standard deviations are less than $30 \%$. 
TABLE 3. ICL and PPC specific activities

\begin{tabular}{lccc}
\hline \multirow{2}{*}{\multicolumn{1}{c}{ Strain/plasmid }} & \multicolumn{3}{c}{ Sp act (nmol/min/mg of protein) of: } \\
\cline { 2 - 3 } & \multicolumn{2}{c}{ ICL at: } & PPC at 11 h \\
\cline { 2 - 3 } & $3 \mathrm{~h}$ & $11 \mathrm{~h}$ & \\
\hline JCL1225/pJF118EH & 2.6 & 3.2 & 33 \\
JCL1225/pPC201 & 2.9 & 3.9 & 210 \\
JCL1535/pJF118EH & 9.4 & 15 & \\
JCL1535/pPC201 & 9.1 & 13 & \\
\hline
\end{tabular}

${ }^{a}$ Samples $(1 \mathrm{ml})$ were taken at the indicated time points and analyzed. All independent measurements are in triplicate, and standard deviations are less than $10 \%$.

ometry defined in Fig. 1 has only three degrees of freedom, which means that the system can be completely determined if three independent fluxes are specified. The number of degrees of freedom can be calculated by using linear algebra techniques. In Fig. 1a, we specified the external fluxes according to experimental data and calculated the internal fluxes. We can also specify three independent internal fluxes and calculate all other fluxes, including the external fluxes. By using this approach, one can evaluate the consequence of manipulating a specific flux relative to glucose consumption based on the stoichiometric model. This approach is called inverse flux analysis and is described in detail elsewhere (8).

We then ask the following question: what is the impact of increasing the anaplerotic flux relative to glucose consumption? To predict the possible outcomes, we specified three fluxes, glucose uptake, the PPC reaction, and respiration, and calculated all other fluxes. The glucose flux is fixed at unity because it is used as a basis for normalization. The PPC flux is specified at a value higher than that calculated from experimental data so as to predict the effect of increasing the anaplerotic flux. The respiration flux is specified at the same level as that calculated from data, because respiration is typically limited by the oxygen transfer rate. By using these three specified fluxes, we calculated all of the relative internal and external fluxes, which are shown in Fig. 1b. Note that by increasing the PPC flux relative to the glucose flux, the demand for precursor metabolites for biosynthesis is increased. Thus, the fraction of carbon flux directed to acetate is greatly reduced. Since the anaplerotic flux can also be achieved through the glyoxylate bypass, Fig. 1c shows that increasing this flux has a similar effect on all other fluxes, particularly the acetate excretion flux.

An increase in the anaplerotic flux relative to glucose consumption can be achieved by increasing the activity of enzymes involved in the anaplerotic reactions, such as PPC and the glyoxylate bypass enzymes. Consistent with the model prediction, we demonstrated experimentally that simultaneously overexpressing PPC or derepressing the glyoxylate bypass indeed reduced the excretion of acetate without compromising other growth characteristics. These results indicate that the excretion of acetate in aerobic cultures is at least partially due to the limitation of anaplerotic fluxes leading to biosynthesis. Additionally, the growth rate of the PPC-overexpressing strains is not substantially affected, suggesting that glucose transport is not limiting growth and that the utilization of the carbon source is more efficient in PPC-overexpressing strains.

Activation of the glyoxylate bypass has been reported to increase acetate utilization during growth on glucose in highcell-density cultures (19). The product of the fadR gene is one of the regulators of the glyoxylate bypass, and fadR mutants have increased constitutive expression of the glyoxylate bypass enzymes (23-25). The fadR mutant strain JCL1535, without
TABLE 4. Concentrations of excreted pyruvate and succinate after $11 \mathrm{~h}$ of growth in glucose minimal medium ${ }^{a}$

\begin{tabular}{lcc}
\hline \multirow{2}{*}{\multicolumn{1}{c}{ Strain/plasmid }} & \multicolumn{2}{c}{ Concn $(\mu \mathrm{M})$ of: } \\
\cline { 2 - 3 } & Pyruvate & Succinate \\
\hline JCL1225/pJF118EH & 10.9 & 0.62 \\
JCL1225/pPC201 & 4.5 & 0.59 \\
JCL1535/pJF118EH & 6.6 & 1.32 \\
JCL1535/pPC201 & 6.6 & 1.1 \\
\hline
\end{tabular}

${ }^{a}$ A $20-\mu l$ sample from each time point was analyzed by high-pressure liquid chromatography. All independent measurements are in triplicate, and standard deviations are less than $30 \%$.

PPC overexpression, shows decreased acetate production. Combining glyoxylate bypass derepression and PPC overexpression results in the lowest acetate excretion. It can be argued that the reduced acetate yield in the fadR strains was due to acetate utilization after excretion. However, since there is no separation of acetate excretion and utilization phases, the reduction of acetate excretion can be attributed to the better utilization of acetyl-CoA throughout the course of the culture. There is no evidence for simultaneous production and utilization of acetate. The fadR strains displayed lower acetate and biomass yields than their isogenic wild-type counterparts. The remaining carbon diverted from acetate apparently goes to $\mathrm{CO}_{2}$ and other fermentation products, such as succinate.

We have overexpressed PPC sevenfold by adding $50 \mu \mathrm{M}$ IPTG and ICL at least threefold by introducing a fadR::Tn10 insertional mutation. According to our flux analysis, however, we only need to increase the anaplerotic flux by about $8 \%$ to decrease acetate excretion by $50 \%$. Apparently, the fluxes through these pathways are tightly controlled or limitation in downstream or other biosynthetic pathways becomes significant after PPC overexpression and glyoxylate bypass derepression. For industrial applications, moderate PPC overexpression can be achieved by using a single copy of the $p p c$ gene expressed from a controllable promoter. Therefore, the approach presented here introduces minimal changes to the host and is easily applicable to industrial strains.

The reduced acetate production demonstrated here raises a question: if acetate excretion can be reduced or avoided, why has $E$. coli not evolved a better control mechanism to minimize acetate excretion? Holms (14) has argued that during the course of E. coli evolution, glucose was not the major carbon source. Instead, fructose and glycerol, for example, were exerting the selection pressure. Thus, E. coli does not excrete acetate aerobically from fructose or glycerol. This argument cannot be tested and is not very convincing, since the biosynthetic efficiency, defined as the ratio between biosynthetic flux and the $\mathrm{CO}_{2}$ flux, of glucose is the highest among all carbon sources (14). Apparently, E. coli has been evolved to optimize glucose metabolism. The second argument of Holms is that acetate excretion is not necessarily inefficient, as the biosynthetic efficiency from glucose is still the highest among all carbon sources. This is true only if different carbon sources are compared. E. coli can achieve a higher biosynthetic efficiency from glucose by increasing the anaplerotic fluxes, as demonstrated here. PPC overexpression leads to higher biosynthetic efficiency without affecting glucose consumption and growth. Additionally, the fadR null mutation decreases acetate production although the utilization of glucose is less efficient than that of the wild type. It is possible that the fadR mutation adversely affects the survival or growth of $E$. coli under conditions rich in fatty acids, since this gene affects other genes involved in fatty 
acid degradation (23-25). Many other mutations can lead to increased anaplerotic flux. For example, reduced or altered allosteric inhibition of PPC or ICL may lead to increased anaplerotic flux without compromising the growth rate or other physiological functions. These possible mutations remain to be selected and tested to further investigate this problem.

\section{ACKNOWLEDGMENTS}

This work was sponsored by the Department of Energy (grant DEFG03-95ER20205) and the Welch Foundation (grant A-1251).

\section{REFERENCES}

1. Aristidou, A. A., K.-Y. San, and G. N. Bennett. 1994. Modification of central metabolic pathway in Escherichia coli to reduce acetate accumulation by heterologous expression of the Bacillus subtilis acetolactate synthase gene. Biotechnol. Bioeng. 44:944-951.

2. Bauer, K. A. A., A. Ben-Bassat, M. Dawson, V. T. de la Puente, and J. O. Neway. 1990. Improved expression of human interleukin-2 in high-cell-density fermentation cultures of Escherichia coli K-12 by a phosphotransacetylase mutant. Appl. Environ. Microbiol. 56:1296-1302.

3. Brice, C. G., and H. L. Kornberg. 1968. Genetic control of isocitrate lyase activity in Escherichia coli. J. Bacteriol. 96:2185-2186.

4. Chao, Y. P., and J. C. Liao. 1993. Alteration of growth yield by overexpression of $p p c$ and pck in Escherichia coli. Appl. Environ. Microbiol. 59:42614265 .

5. Chin, A. M., D. A. Feldheim, and M. H. Saier, Jr. 1989. Altered transcriptional patterns affecting several metabolic pathways in strains of Salmonella typhimurium which overexpress the fructose regulon. J. Bacteriol. 171:24242434.

6. Chou, C.-H., G. N. Bennett, and K.-Y. San. 1994. Effect of modified glucose uptake using genetic engineering techniques on high-level recombinant protein production in Escherichia coli dense cultures. Biotechnol. Bioeng. 44: 952-960.

7. Dedhia, N. N., T. Hottinger, and J. E. Bailey. 1994. Overproduction of glycogen in Escherichia coli blocked in the acetate pathway improves cell growth. Biotechnol. Bioeng. 44:132-139.

8. Delgado, J., and J. C. Liao. Inverse flux analysis for reduction of acetate excretion in Escherichia coli. Biotechnol. Prog., in press.

9. Diaz-Ricci, J. C., L. Regan, and J. E. Bailey. 1991. Effect of alteration of the acetic acid synthesis pathway on the fermentation pattern of Escherichia coli. Biotechnol. Bioeng. 38:1318-1324.

10. Dixon, G. H., and H. L. Kornberg. 1959. Assay methods for key enzymes of the glyoxylate cycle. Biochem. J. 72:1-3.

11. El-Mansi, E. M. T., and W. H. Holms. 1989. Control of carbon flux to acetate excretion during growth of $E$. coli in batch and continuous culture. J. Gen. Microbiol. 135: 2875-2883.

12. Furste, J. P., W. Pansegrau, R. Frank, H. Blocker, P. Scholz, M. Bagdasarian, and E. Lanka. 1986. Molecular cloning of the plasmid RP4 primase region in a multi-host-range $t a c P$ expression vector. Gene 48:119-131.

13. Goldie, A. H., and B. D. Sanwal. 1980. Genetic and physiological characterization of Escherichia coli mutants deficient in phosphoenolpyruvate carboxykinase activity. J. Bacteriol. 141:1115-1121.

14. Holms, W. H. 1986. The central metabolic pathways in Escherichia coli: relationship between flux and control at a branchpoint, efficiency of conversion to biomass, and excretion of acetate. Curr. Top. Cell. Regul. 28:69-105.

15. Iuchi, S., D. C. Cameron, and E. C. C. Lin. 1989. A second global regulator gene $(\operatorname{arc} B)$ mediating repression of enzymes in aerobic pathways in Esche- richia coli. J. Bacteriol. 171:868-873.

16. Iuchi, S., and E. C. C. Lin. 1988. $\operatorname{arc} A$ (dye), a global regulatory gene in Escherichia coli mediating repression of enzymes in aerobic pathways. Proc. Natl. Acad. Sci. USA 85:1888-1892.

17. Kleman, G. L., J. J. Chalmers, G. W. Luli, and W. R. Strohl. 1991. A predictive and feedback control algorithm maintains a constant glucose concentration in fed-batch fermentations. Appl. Environ. Microbiol. 57:910 917.

18. Kleman, G. L., J. L. Chalmers, G. W. Luli, and W. R. Strohl. 1991. Glucosestat, a glucose-controlled continuous culture. Appl. Environ. Microbiol. 57 918-923.

19. Kleman, G. L., and W. R. Strohl. 1994. Acetate metabolism by Escherichia coli in high-cell-density fermentation. Appl. Environ. Microbiol. 60:39523958

20. Kornberg, H. 1966. The role and control of the glyoxylate cycle in Escherichia coli. Biochem. J. 99:1-11.

21. Landwall, P., and T. Holme. 1977. Influence of glucose and dissolved oxygen concentrations on yields of Escherichia coli B in dialysis culture. J. Gen. Microbiol. 103:353-358.

22. LaPorte, D. C., K. Walsh, and D. E. Koshland, Jr. 1984. The branch point effect. J. Biol. Chem. 259:14068-14075.

23. Maloy, S. R., M. Bohlander, and W. D. Nunn. 1980. Elevated levels of glyoxylate shunt enzymes in Escherichia coli strains constitutive for fatty acid degradation. J. Bacteriol. 143:720-725.

24. Maloy, S. R., and W. D. Nunn. 1982. Genetic regulation of the glyoxylate shunt in Escherichia coli K-12. J. Bacteriol. 149:173-180.

25. Maloy, S. R., and W. D. Nunn. 1995. Role of gene fadR in Escherichia coli acetate metabolism. J. Bacteriol. 148:83-90.

26. Miller, G. L. 1958. Use of dinitrosalicylic acid reagent for determination of reducing sugars. Anal. Chem. 31:426-428.

27. Miller, J. H. 1972. Experiments in molecular genetics. Cold Spring Harbor Laboratory, Cold Spring Harbor, N.Y.

28. Mori, H., T. Yano, T. Kobayashi, and S. Shimizu. 1979. High density cultivation of biomass in fed-batch system with DO-stat. J. Chem. Eng. Jpn. 12:313-319.

29. Morikawa, M., K. Izui, M. Taguchi, and H. Katsuki. 1980. Regulation of Escherichia coli phosphoenolpyruvate carboxylase by multiple effectors in vivo. I. Estimation of the activities in the cells grown on various compounds. J. Biochem. 87:441-449.

30. Paalme, T., K. Tiisma, A. Kahru, K. Vanatalu, and R. Vilu. 1990. Glucoselimited fed-batch cultivation of Escherichia coli with computer-controlled fixed growth rate. Biotechnol. Bioeng. 35:312-319.

31. Patnaik, R., W. D. Roof, R. F. Young, and J. C. Liao. 1992. Stimulation of glucose catabolism in Escherichia coli by a potential futile cycle. J. Bacteriol. 174:7527-7532.

32. Reiling, H. E., H. Laurila, and A. Fiechter. 1985. Mass culture of Escherichia coli: medium development for low and high density cultivation of Escherichia coli $\mathrm{B} / \mathrm{r}$ in minimal and complex media. J. Biotechnol. 2:191-206.

33. Shiloach, J., J. Kaufman, A. S. Guillard, and R. Fass. 1996. Effect of glucose supply strategy on acetate accumulation, growth, and recombinant protein production by Escherichia coli BL21 ( $\mathrm{DDE} 3$ ) and Escherichia coli JM109. Biotechnol. Bioeng. 49:421-428.

34. Stephanopoulos, G., and J. J. Vallino. 1991. Network rigidity and metabolic engineering in metabolite overproduction. Science 252:1675-1681.

35. Sunnarborg, A., D. Klump, T. Chung, and D. C. LaPorte. 1990. Regulation of the glyoxylate bypass operon: cloning and characterization of $i c l R$. J. Bacteriol. 163:2642-2649.

36. Vallino, J. J., and G. Stephanopoulos. 1993. Metabolic flux distributions in Corynebacterium glutamicum during growth and lysine overproduction. Biotechnol. Bioeng. 41:633-646. 